\title{
Quantification of Death Risk in Relation to Sex, Pre-Existing Cardiovascular Diseases and Risk Factors in COVID-19 Patients: Let's Take Stock and See Where We Are
}

\author{
Amalia Ioanna Moula ${ }^{1,+} \oplus$, Linda Renata Micali ${ }^{1,+}$, Francesco Matteucci ${ }^{1}$, Fabiana Lucà ${ }^{2}$, \\ Carmelo Massimiliano Rao ${ }^{2}$, Orlando Parise ${ }^{1}$ (1), Gianmarco Parise ${ }^{1}$, \\ Michele Massimo Gulizia ${ }^{3,4}$ and Sandro Gelsomino ${ }^{1, * \mathbb{D}}$ \\ 1 Department of Cardiothoracic Surgery, Cardiovascular Research Institute Maastricht University, \\ Universiteitssingel 50, 6229 ER Maastricht, The Netherlands; amaliamoula1@gmail.com (A.I.M.); \\ 1.micali@maastrichtuniversity.nl (L.R.M.); francesco.matteucci@maastrichtuniversity.nl (F.M.); \\ o.parise@icloud.com (O.P.); g.parise@maastrichtuniversity.nl (G.P.) \\ 2 Grande Ospedale Metropolitano, 89124 Reggio Calabria, Italy; fabiana.luca92@gmail.com (F.L.); \\ massimo.rao@libero.it (C.M.R.) \\ 3 Cardiogy Complex Unit, Garibaldi Nesima Hospital, 95122 Catania, Italy; michele.gulizia60@gmail.com \\ 4 Heart Care Foundation Onlus, 50121 Florence, Italy \\ * Correspondence: sandro.gelsomino@maastrichtuniveristy.nl \\ + Amalia Ioanna Moula and Linda Renata Micali equally contributed to this work.
}

Received: 6 July 2020; Accepted: 17 August 2020; Published: 19 August 2020

check for updates

\begin{abstract}
Patients with pre-existing cardiovascular disease (CVD) might be more susceptible to infection from severe acute respiratory syndrome coronavirus 2 (SARS-CoV-2) and have higher mortality rates. Nevertheless, the risk of mortality has not been previously quantified. The aim of this meta-analysis is to quantify the risk of mortality in coronavirus disease 2019 (COVID-19) patients. A meta-analysis was conducted analyzing the impact of (1) sex, (2) age, (3) CVD with coronary artery disease (CAD), (4) CAD alone, (5) CVD without CAD, (6) hypertension, (7) cerebrovascular diseases, and (8) diabetes on mortality. Relative risk was assessed for dichotomous variables, mean difference for continuous variables. Twenty-six studies were included, encompassing 8497 patients. Males had $16 \%$ higher risk of mortality than females $(p<0.05)$ and elderly patients had higher chance of dying than younger patients $(p<0.0001)$. Patients with overall CVD have a 1.96 -fold higher mortality risk $(p<0.0001)$. CAD increases risk of mortality by 1.90 -fold $(p<0.05)$. CVD-CAD were found to increase risk up to 2.03-fold $(p<0.05)$. Hypertension, cerebrovascular disease and diabetes increase the risk of death up to 1.73-fold, 1.76-fold and 1.59-fold, respectively $(p<0.0001, p<0.0001, p<0.05$, respectively). Sex, age, presence of CAD and/or other types of CVD, hypertension, cerebrovascular diseases and diabetes mellitus increase mortality in patients with COVID-19.
\end{abstract}

Keywords: COVID-19; SARS-CoV-2; cardiovascular diseases; mortality; hypertension; diabetes mellitus

\section{Introduction}

Coronavirus disease 2019 (COVID-19) is an infectious disease caused by severe acute respiratory syndrome coronavirus 2 (SARS-CoV-2). The virus, first identified in 2019 in China, has a positive sense single stranded RNA and seems to be of zoonotic origin. The virus is most likely airborne and highly contagious. It spreads via contaminated droplets that pass from one human to another while in close contact [1]. 
The recent global pandemic ignited by the COVID-19 has had a considerable impact on many healthcare systems around the world [2]. For this reason, the disease has received increasing attention by the scientific community. Previous literature suggests that patients with pre-existing cardiovascular disease (CVD) might be potentially more susceptible to infection from SARS-CoV-2 [3]. Nevertheless, the exact mechanisms by which COVID-19 affects the cardiovascular system and mortality are not yet well understood, despite accumulating evidence that such a connection exists $[4,5]$.

However, to the best of our knowledge the risk of death in relation to sex, age and CVD has not been quantified in large cohorts of patients. Moreover, the association between COVID-19, CVD and patient mortality has not yet been fully elucidated, setting the need for additional confirmation of the association between these two ailments as well as the impact of shared risk factors on such a relation. Therefore, the present meta-analysis is aimed to quantify the risk of mortality in relation to sex, age and pre-existing CVD in COVID-19 patients, and attempt to identify the potential factors involved in such a causation.

\section{Materials and Methods}

\subsection{Search Strategy}

The literature search was conducted in accordance with the principles of the Preferred Reporting Items for Systematic Reviews and Meta-Analyses (PRISMA) [6] and the Cochrane handbook [7]. Two authors established the search strategy (AIM and LRM) and the decisions were approved by a third author (SG).

One investigator performed the literature search (AIM), which was limited to articles published from 1 December 2019 until 18 May 2020. An unrestricted literature search was performed using PubMed, adopting the following search terms: "severe acute respiratory syndrome coronavirus 2" [Supplementary Concept] OR "COVID-19" [Supplementary Concept] OR "spike glycoprotein, COVID-19 virus" [Supplementary Concept] OR "covid-19" OR "covid 19" OR "COVID19" OR "SARS-CoV-2" OR "novel coronavirus" AND epidemiology OR comorbidities OR heart OR cardiovascular OR myocardial OR "Cardiovascular Diseases" [Mesh] OR heart OR myocardium OR STEMI OR infarction OR arrhythmia OR hypertension.

\subsection{Selection Process}

The articles were selected based on the following inclusion criteria: (1) human studies; (2) full articles about COVID-19 disease containing separate data for patients that survived and patients that did not; (3) analyses of fatality cases; (4) studies including at least 10 patients; (5) articles published from December 2019 and (6) articles in English language.

The exclusion criteria used to reject articles were: (1) non-human studies; (2) case reports; (3) previous reviews and/or meta-analyses; (4) editorials; (5) comments; (6) studies without separate data on surviving and non-surviving patients; (7) studies in languages other than English.

\subsection{Risk of Bias Assessment}

Two reviewers (AM and LM) independently assessed the risk of bias for the included studies. The ROBINS-I tool (Risk of Bias in No-Randomized Studies of Interventions) was used for the assessment of bias at the individual study level [7]. Disagreements were resolved by discussion or by involving a third reviewer (SG). The domains assessed were (1) bias due to confounding; (2) bias in selection of participants into the study; (3) bias in classification of interventions; (4) bias due to deviations from intended interventions; (5) bias due to missing data; (6) bias in measurement of outcomes; (7) bias in selection of the reported result; and (8) overall bias assessment. The evaluation of the aforementioned domains was conducted with the aid of Cochrane handbook [7]. Furthermore, the generation of the plot for ROBINS-I was achieved the software robvis [8]. 


\subsection{Endpoints}

The primary endpoint of this meta-analysis was to identify comorbidities and pre-existing cardio-metabolic diseases that could predict mortality in patients with COVID-19. The risk factors and comorbidities taken into consideration were: (1) sex, (2) age, (3) CVD, (4) coronary artery disease (CAD), (5) hypertension, (6) cerebrovascular disease, and (7) diabetes mellitus.

To evaluate the impact of the several types of CVD and CAD separately, first we conducted an analysis of patients with CVD including CAD ("CVD with CAD") vs. patients without CVD; then a separate analysis only of patients with $\mathrm{CAD}$ ("CAD") vs. patients without $\mathrm{CAD}$; and finally, an analysis of patients with CVD excluding CAD (“CVD without CAD") vs. patients without CVD.

\subsection{Statistical Analysis}

The meta-analysis was conducted using v. 3.6.1 (R Foundation for Statistical Computing, Vienna, Austria). Relative risk (RR) and 95\% confidence interval (CI) were used as index statistics for dichotomous variables. For continuous variables, mean difference and $95 \% \mathrm{CI}$ were calculated. In both cases the random effects model was adopted because heterogeneity among studies was anticipated. Heterogeneity was assessed with the statistical inconsistency Higgin's $\mathrm{I}^{2}$ test [7]. $\mathrm{I}^{2}$ values $<40 \%$ were considered having low heterogeneity, I2 values $>75 \%$ were considered having high heterogeneity [7]. Publication bias was evaluated using Egger's test of the intercept. $p$ values $<0.05$ were considered statistically significant.

\section{Results}

\subsection{Search Results and Characteristics of the Studies}

The initial search retrieved 1719 articles. After screening for the inclusion and exclusion criteria, 78 articles that included patient demographic data for COVID-19 and mortality were found. After rejecting articles without separate data for patients that survived and non-survivors, a total of 22 articles were found to fulfil the criteria. Four additional articles were added from the references of the articles found through the search on PubMed (Figure 1). At the end of the selection process, 26 studies were included in the analysis [9-34]. Eight out of the twenty-six studies were analyses of fatality cases $[11,12,15,18,21,26,33,34]$. There were twenty papers from China [9-16,18,22-32], two papers from Italy $[17,21]$, two papers from Korea [33,34], one from Iran [20] and one from the United States of America [19].

In total, the patient cohort included of 8497 patients, of whom $5121(60.3 \%)$ were male and 3376 $(39.7 \%)$ were female. Non-survivors among males accounted for $25.0 \%$ of the patients (1280 individuals out of 5121), whereas non-survivors percentage among females was 19.5\% (659 individuals out of 3376). The studies that were included in the meta-analysis and the characteristics of the patients with COVID-19 are shown in Table 1. 


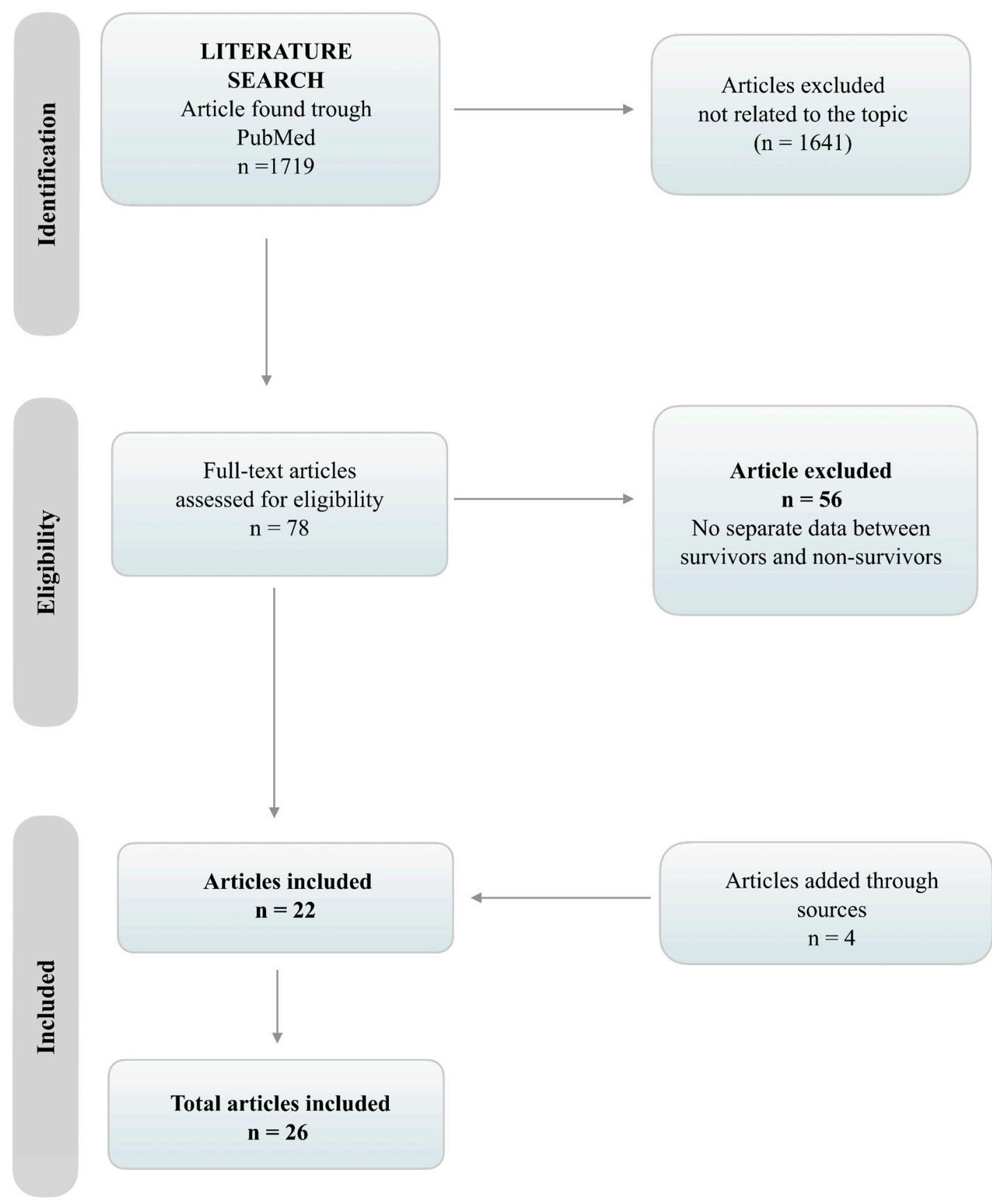

Figure 1. Preferred Reporting Items for Systematic Reviews and Meta-Analyses (PRISMA) flowchart of the selection process. 
Table 1. Patients' baseline characteristics ${ }^{1}$

\begin{tabular}{|c|c|c|c|c|c|c|c|c|c|c|c|c|c|c|c|c|c|c|}
\hline \multirow[t]{2}{*}{ Author, Year } & \multirow[t]{2}{*}{$\begin{array}{c}\text { Study } \\
\text { Design }\end{array}$} & \multicolumn{3}{|c|}{ No. of Patients } & \multicolumn{2}{|c|}{ F/M } & \multicolumn{2}{|c|}{ Age } & \multicolumn{2}{|c|}{ CVD } & \multicolumn{2}{|c|}{ CAD } & \multicolumn{2}{|c|}{ Hypertension } & \multicolumn{2}{|c|}{ Diabetes } & \multicolumn{2}{|c|}{$\begin{array}{c}\text { Cerebrovascular } \\
\text { Disease }\end{array}$} \\
\hline & & Overall & $\mathrm{S}$ & NS & S & NS & $\mathbf{S}$ & NS & $\mathrm{s}$ & NS & $\mathrm{S}$ & NS & $\mathrm{S}$ & NS & $\mathrm{S}$ & NS & $\mathrm{S}$ & NS \\
\hline $\begin{array}{c}\text { Onder et al., } 2020 \\
\text { [21] }\end{array}$ & - & 355 & $0(0)$ & $\begin{array}{c}355 \\
(100)\end{array}$ & 0 & $106 / 249$ & - & $79.5 \pm 8.1$ & $0(0)$ & $\begin{array}{l}117 \\
(33)\end{array}$ & $0(0)$ & $\begin{array}{l}117 \\
(33)\end{array}$ & - & - & $0(0)$ & $\begin{array}{c}126 \\
(35.5)\end{array}$ & $0(0)$ & $34(9.6)$ \\
\hline $\begin{array}{c}\text { Zhou et al., } 2020 \\
\text { [29] }\end{array}$ & MRCS & 191 & $\begin{array}{c}137 \\
(71.7)\end{array}$ & $\begin{array}{c}54 \\
(28.3)\end{array}$ & $56 / 81$ & $16 / 38$ & $\begin{array}{c}52.0 \\
(45.0-58.0)\end{array}$ & $\begin{array}{c}69.0 \\
(63.0-76.0)\end{array}$ & $2(1.5)$ & $\begin{array}{c}13 \\
(24.1)\end{array}$ & $2(1.5)$ & $\begin{array}{c}13 \\
(24.1)\end{array}$ & $\begin{array}{c}32 \\
(23.4)\end{array}$ & $\begin{array}{c}26 \\
(48.2)\end{array}$ & $\begin{array}{c}19 \\
(13.9)\end{array}$ & $\begin{array}{c}17 \\
(31.5)\end{array}$ & - & - \\
\hline $\begin{array}{c}\text { Chen et al., } 2020 \text { * } \\
\text { [11] }\end{array}$ & $\mathrm{RCsSr}$ & 274 & $\begin{array}{c}161 \\
(58.8)\end{array}$ & $\begin{array}{l}113 \\
(41.2)\end{array}$ & $73 / 88$ & $30 / 83$ & $\begin{array}{c}51.0 \\
(37.0-66.0)\end{array}$ & $\begin{array}{c}68.0 \\
(62.0-77.0)\end{array}$ & $7(4.3)$ & $\begin{array}{c}16 \\
(14.2)\end{array}$ & - & - & $\begin{array}{c}39 \\
(24.2)\end{array}$ & $\begin{array}{c}54 \\
(47.8)\end{array}$ & $\begin{array}{c}23 \\
(14.3)\end{array}$ & $\begin{array}{c}24 \\
(21.3)\end{array}$ & $0(0)$ & $4(3.5)$ \\
\hline $\begin{array}{c}\text { Deng et al., } 2020 \\
\text { [13] }\end{array}$ & RCS & 225 & $\begin{array}{l}116 \\
(51.6)\end{array}$ & $\begin{array}{c}109 \\
(48.4)\end{array}$ & $65 / 51$ & $36 / 73$ & $\begin{array}{c}40.0 \\
(33.0-57.0)\end{array}$ & $\begin{array}{c}69.0 \\
(62.0-74.0)\end{array}$ & $4(3.4)$ & $\begin{array}{c}13 \\
(11.9)\end{array}$ & $4(3.4)$ & $\begin{array}{c}13 \\
(11.9)\end{array}$ & $\begin{array}{c}18 \\
(15.5)\end{array}$ & $\begin{array}{c}40 \\
(36.7)\end{array}$ & $9(7.8)$ & $\begin{array}{c}17 \\
(15.6)\end{array}$ & - & - \\
\hline $\begin{array}{c}\text { Yuan et al., } 2020 \\
\text { [28] }\end{array}$ & RCS & 27 & $\begin{array}{c}17 \\
(63.0)\end{array}$ & $\begin{array}{c}10 \\
(37.0)\end{array}$ & $9 / 8$ & $6 / 4$ & $\begin{array}{c}55.0 \\
(35.0-60.0)\end{array}$ & $\begin{array}{c}68.0 \\
(63.0-73.0)\end{array}$ & $0(0)$ & $\begin{array}{c}3 \\
(30.0)\end{array}$ & $0(0)$ & $\begin{array}{c}3 \\
(30.0)\end{array}$ & $0(0)$ & $\begin{array}{c}5 \\
(50.0)\end{array}$ & $0(0)$ & $\begin{array}{c}6 \\
(60.0)\end{array}$ & $0(0)$ & $1(10.0)$ \\
\hline $\begin{array}{c}\text { Yang et al., } 2020^{*} \\
\text { [27] }\end{array}$ & SROS & 52 & $\begin{array}{c}20 \\
(38.5)\end{array}$ & $\begin{array}{c}32 \\
(61.5)\end{array}$ & $6 / 14$ & $11 / 21$ & $51.9 \pm 12.9$ & $64.6 \pm 11.2$ & $\begin{array}{c}2 \\
(10.0)\end{array}$ & $3(9.4)$ & $\begin{array}{c}2 \\
(10.0)\end{array}$ & $3(9.4)$ & - & - & $\begin{array}{c}2 \\
(10.0)\end{array}$ & $\begin{array}{c}7 \\
(21.9)\end{array}$ & $0(0)$ & $7(21.9)$ \\
\hline $\begin{array}{l}\text { Jeong et al., } 2020 \\
\text { (KCDCP) [33] }\end{array}$ & & 66 & $0(0)$ & $\begin{array}{l}66 \\
(100)\end{array}$ & 0 & $29 / 37$ & - & 77 (35-93) & $0(0)$ & $\begin{array}{c}10 \\
(15.2)\end{array}$ & $0(0)$ & $\begin{array}{c}10 \\
(15.2)\end{array}$ & $0(0)$ & $\begin{array}{c}30 \\
(45.5)\end{array}$ & $0(0)$ & $\begin{array}{c}23 \\
(34.8)\end{array}$ & $0(0)$ & $5(7.6)$ \\
\hline $\begin{array}{l}\text { Park et al., } 2020 \\
\text { (KSID) [34] }\end{array}$ & & 54 & $0(0)$ & $\begin{array}{c}54 \\
(100)\end{array}$ & - & - & - & - & - & - & - & - & - & - & - & $\begin{array}{c}16 \\
(29.6)\end{array}$ & - & - \\
\hline Cao et al., 2020 [9] & - & 102 & $\begin{array}{c}85 \\
(83.3)\end{array}$ & $\begin{array}{c}17 \\
(16.7)\end{array}$ & $45 / 40$ & $4 / 13$ & $\begin{array}{c}53.0 \\
(47.0-66.0)\end{array}$ & $\begin{array}{c}72.0 \\
(63.0-81.0)\end{array}$ & $2(2.4)$ & $\begin{array}{c}3 \\
(17.6)\end{array}$ & - & - & $\begin{array}{c}17 \\
(20.0)\end{array}$ & $\begin{array}{c}11 \\
(64.7)\end{array}$ & $5(5.9)$ & $\begin{array}{c}6 \\
(35.3)\end{array}$ & $3(3.5)$ & $3(17.6)$ \\
\hline $\begin{array}{c}\text { Wang et al., } 2020 * \\
{[25]}\end{array}$ & RCS & 339 & $\begin{array}{c}274 \\
(80.2)\end{array}$ & $\begin{array}{c}65 \\
(19.8)\end{array}$ & $147 / 127$ & $26 / 39$ & $\begin{array}{c}68.0 \\
(64.0-74.0)\end{array}$ & $\begin{array}{c}76.0 \\
(70.0-83.0)\end{array}$ & $\begin{array}{c}32 \\
(11.7)\end{array}$ & $\begin{array}{c}21 \\
(32.3)\end{array}$ & - & - & $\begin{array}{l}106 \\
(38.7)\end{array}$ & $\begin{array}{c}32 \\
(49.2)\end{array}$ & $\begin{array}{c}43 \\
(15.7)\end{array}$ & $\begin{array}{c}11 \\
(16.9)\end{array}$ & $\begin{array}{c}11 \\
(4.0)\end{array}$ & $\begin{array}{c}10 \\
(15.4)\end{array}$ \\
\hline $\begin{array}{c}\text { Du et al., } 2020 * \\
\text { [15] }\end{array}$ & ROS & 85 & $0(0)$ & $\begin{array}{c}85 \\
(100)\end{array}$ & 0 & $23 / 62$ & 50 & $65.8 \pm 14.2$ & $0(0)$ & $\begin{array}{c}10 \\
(11.8)\end{array}$ & $0(0)$ & $\begin{array}{c}10 \\
(11.8)\end{array}$ & $0(0)$ & $\begin{array}{c}32 \\
(37.6)\end{array}$ & $0(0)$ & $\begin{array}{c}19 \\
(22.4)\end{array}$ & $0(0)$ & $7(8.2)$ \\
\hline $\begin{array}{c}\text { Du et al., } 2020^{*} \\
{[14]}\end{array}$ & PCS & 179 & $\begin{array}{c}158 \\
(88.3)\end{array}$ & $\begin{array}{c}21 \\
(11.7)\end{array}$ & $71 / 87$ & $11 / 10$ & $56.0 \pm 13.5$ & $70.2 \pm 7.7$ & $\begin{array}{c}17 \\
(10.8)\end{array}$ & $\begin{array}{c}12 \\
(57.1)\end{array}$ & - & - & $\begin{array}{c}45 \\
(28.5)\end{array}$ & $\begin{array}{c}13 \\
(61.9)\end{array}$ & $\begin{array}{c}27 \\
(17.1)\end{array}$ & $\begin{array}{c}6 \\
(28.6)\end{array}$ & - & - \\
\hline $\begin{array}{c}\text { Chen et al., 2020 * } \\
\text { [10] }\end{array}$ & RCS & 1590 & $\begin{array}{l}1540 \\
(96.9)\end{array}$ & $\begin{array}{c}50 \\
(3.1)\end{array}$ & $675 / 865$ & $20 / 30$ & $\begin{array}{c}48.0 \\
(1.0-94.0) \dagger\end{array}$ & $\begin{array}{c}69.0 \\
(51.0-86.0) \\
+\end{array}$ & $\begin{array}{c}51 \\
(3.3)\end{array}$ & $\begin{array}{c}8 \\
(16.0)\end{array}$ & $\begin{array}{c}51 \\
(3.3)\end{array}$ & $\begin{array}{c}8 \\
(16.0)\end{array}$ & $\begin{array}{c}241 \\
(15.6)\end{array}$ & $\begin{array}{c}28 \\
(56.0)\end{array}$ & $\begin{array}{l}117 \\
(7.6)\end{array}$ & $\begin{array}{c}13 \\
(26.0)\end{array}$ & $\begin{array}{c}24 \\
(1.6)\end{array}$ & $6(12.0)$ \\
\hline Li et al., 2020 [18] & ROS & 25 & $0(0)$ & $\begin{array}{c}25 \\
(100)\end{array}$ & 0 & $15 / 10$ & - & $\begin{array}{c}73.0 \\
(55.0-100.0)\end{array}$ & $0(0)$ & $\begin{array}{c}8 \\
(32.0)\end{array}$ & - & - & $0(0)$ & $\begin{array}{c}16 \\
(64.0)\end{array}$ & $0(0)$ & $\begin{array}{c}10 \\
(40.0)\end{array}$ & $0(0)$ & $4(16.0)$ \\
\hline $\begin{array}{c}\text { Yang et al., } 2020 \text { * } \\
\text { [26] }\end{array}$ & ROS & 92 & $0(0)$ & $\begin{array}{c}92 \\
(100)\end{array}$ & 0 & $43 / 49$ & - & $\begin{array}{l}69.8 \pm 14.5 \\
(30.0-97.0)\end{array}$ & $0(0)$ & $\begin{array}{c}16 \\
(17.4)\end{array}$ & - & - & $0(0)$ & $\begin{array}{c}51 \\
(55.4)\end{array}$ & $0(0)$ & $\begin{array}{c}13 \\
(14.1)\end{array}$ & - & - \\
\hline $\begin{array}{c}\text { Wang et al., } 2020 * \\
\text { [23] }\end{array}$ & $\mathrm{RCsSr}$ & 107 & $\begin{array}{c}88 \\
(82.2)\end{array}$ & $\begin{array}{c}19 \\
(17.8)\end{array}$ & $47 / 41$ & $3 / 16$ & $\begin{array}{c}44.5 \\
(35.0-58.8)\end{array}$ & $\begin{array}{c}73.0 \\
(64.0-81.0)\end{array}$ & $6(6.8)$ & $\begin{array}{c}7 \\
(36.8)\end{array}$ & - & - & $\begin{array}{c}16 \\
(18.2)\end{array}$ & $\begin{array}{c}10 \\
(52.6)\end{array}$ & $6(6.8)$ & $\begin{array}{c}5 \\
(26.3)\end{array}$ & $3(3.4)$ & $3(15.8)$ \\
\hline $\begin{array}{l}\text { Nikpouraghdam et } \\
\text { al., } 2020[20]\end{array}$ & SRCS & 2964 & $\begin{array}{l}2725 \\
(91.9)\end{array}$ & $\begin{array}{l}239 \\
(8.1)\end{array}$ & $937 / 1788$ & $72 / 167$ & - & $\begin{array}{c}65.0 \\
(57.0-75.0)\end{array}$ & $\begin{array}{c}33 \\
(1.2)\end{array}$ & $4(1.7)$ & - & - & $\begin{array}{l}51 \\
(1.9)\end{array}$ & $8(3.3)$ & $\begin{array}{c}102 \\
(3.7)\end{array}$ & $\begin{array}{c}11 \\
(4.6)\end{array}$ & - & - \\
\hline $\begin{array}{c}\text { Mehta et al., } 2020 \\
\text { [19] }\end{array}$ & SROS & 218 & $\begin{array}{c}157 \\
(72.0)\end{array}$ & $\begin{array}{c}61 \\
(28.0)\end{array}$ & $66 / 91$ & $25 / 36$ & $\begin{array}{c}66.0 \\
(10.0-92.0)\end{array}$ & $\begin{array}{c}76.0 \\
(10.0-92.0)\end{array}$ & $\begin{array}{c}42 \\
(26.8)\end{array}$ & $\begin{array}{c}34 \\
(55.7)\end{array}$ & $\begin{array}{c}24 \\
(15.3)\end{array}$ & $\begin{array}{c}19 \\
(31.1)\end{array}$ & $\begin{array}{c}100 \\
(63.7)\end{array}$ & $\begin{array}{c}47 \\
(77.0)\end{array}$ & $\begin{array}{c}53 \\
(33.8)\end{array}$ & $\begin{array}{c}27 \\
(44.3)\end{array}$ & - & - \\
\hline
\end{tabular}


Table 1. Cont.

\begin{tabular}{|c|c|c|c|c|c|c|c|c|c|c|c|c|c|c|c|c|c|c|}
\hline \multirow[t]{2}{*}{ Author, Year } & \multirow[t]{2}{*}{$\begin{array}{l}\text { Study } \\
\text { Design }\end{array}$} & \multicolumn{3}{|c|}{ No. of Patients } & \multicolumn{2}{|c|}{ F/M } & \multicolumn{2}{|c|}{ Age } & \multicolumn{2}{|c|}{ CVD } & \multicolumn{2}{|c|}{ CAD } & \multicolumn{2}{|c|}{ Hypertension } & \multicolumn{2}{|c|}{ Diabetes } & \multicolumn{2}{|c|}{$\begin{array}{l}\text { Cerebrovascular } \\
\text { Disease }\end{array}$} \\
\hline & & Overall & $S$ & NS & $S$ & NS & $\mathrm{S}$ & NS & $\mathrm{S}$ & NS & $\mathrm{S}$ & NS & $\mathrm{S}$ & NS & $\mathrm{S}$ & NS & $\mathrm{S}$ & NS \\
\hline $\begin{array}{c}\text { Wang et al., 2020a } \\
{ }^{*} \ddagger[24]\end{array}$ & - & 296 & $\begin{array}{c}277 \\
(93.6)\end{array}$ & $\begin{array}{c}19 \\
(6.4)\end{array}$ & $148 / 129$ & $8 / 11$ & $46.0 \pm 14.4$ & $65.6 \pm 12.6$ & $5(1.8)$ & $\begin{array}{c}5 \\
(26.3)\end{array}$ & $5(1.8)$ & $\begin{array}{c}5 \\
(26.3)\end{array}$ & $\begin{array}{c}33 \\
(11.9)\end{array}$ & $\begin{array}{c}9 \\
(47.4)\end{array}$ & $\begin{array}{c}24 \\
(8.7)\end{array}$ & $\begin{array}{c}6 \\
(31.6)\end{array}$ & $4(1.4)$ & $3(15.8)$ \\
\hline $\begin{array}{c}\text { Wang et al., } 2020 \mathrm{~b} \\
{ }^{*} \ddagger[24]\end{array}$ & - & 44 & $\begin{array}{c}30 \\
(68.2)\end{array}$ & $\begin{array}{c}14 \\
(31.8)\end{array}$ & $16 / 14$ & $4 / 10$ & $48.8 \pm 14.2$ & $69.0 \pm 13.4$ & $0(0)$ & $\begin{array}{c}3 \\
(21.4)\end{array}$ & $0(0)$ & $\begin{array}{c}3 \\
(21.4)\end{array}$ & $\begin{array}{c}7 \\
(23.3)\end{array}$ & $\begin{array}{c}4 \\
(28.6)\end{array}$ & $\begin{array}{c}5 \\
(16.7)\end{array}$ & $\begin{array}{c}4 \\
(28.6)\end{array}$ & $1(3.3)$ & $1(7.1)$ \\
\hline $\begin{array}{c}\text { Chen et al., 2020 * } \\
\text { [12] }\end{array}$ & RCS & 38 & $0(0)$ & $\begin{array}{c}38 \\
(100)\end{array}$ & 0 & $11 / 27$ & - & $\begin{array}{c}70.0 \\
(36.0-89.0)\end{array}$ & $0(0)$ & $\begin{array}{c}5 \\
(13.2)\end{array}$ & $0(0)$ & $\begin{array}{c}5 \\
(13.2)\end{array}$ & $0(0)$ & $\begin{array}{c}15 \\
(39.5)\end{array}$ & $00(0)$ & $\begin{array}{c}11 \\
(28.9)\end{array}$ & $0(0)$ & $4(10.5)$ \\
\hline $\begin{array}{c}\text { Inciardi et al., } 2020 \\
\text { [17] }\end{array}$ & - & 53 & $\begin{array}{c}34 \\
(64.2)\end{array}$ & $\begin{array}{c}19 \\
(35.8)\end{array}$ & $6 / 28$ & $2 / 17$ & $65.5 \pm 10.2$ & $72.3 \pm 13.5$ & $\begin{array}{c}34 \\
(100)\end{array}$ & $\begin{array}{c}19 \\
(100)\end{array}$ & - & - & $\begin{array}{c}27 \\
(79.4)\end{array}$ & $\begin{array}{c}13 \\
(68.4)\end{array}$ & $\begin{array}{c}7 \\
(20.0)\end{array}$ & $\begin{array}{c}9 \\
(47.4)\end{array}$ & - & - \\
\hline Sun et al., 2020 [22] & $\mathrm{RCC}$ & 244 & $\begin{array}{l}123 \\
(50.4)\end{array}$ & $\begin{array}{c}121 \\
(49.6)\end{array}$ & $72 / 51$ & $39 / 82$ & $\begin{array}{c}67.0 \\
(64.0-72.0)\end{array}$ & $\begin{array}{c}72.0 \\
(66.0-78.0)\end{array}$ & $\begin{array}{c}15 \\
(12.9)\end{array}$ & $\begin{array}{c}20 \\
(16.5)\end{array}$ & $\begin{array}{c}15 \\
(12.9)\end{array}$ & $\begin{array}{c}20 \\
(16.5)\end{array}$ & $\begin{array}{c}62 \\
(50.4)\end{array}$ & $\begin{array}{c}76 \\
(62.8)\end{array}$ & $\begin{array}{c}24 \\
(19.5)\end{array}$ & $\begin{array}{c}27 \\
(22.3)\end{array}$ & - & - \\
\hline $\begin{array}{c}\text { Huang et al., } 2020 \\
\text { [16] }\end{array}$ & RCS & 299 & $\begin{array}{c}283 \\
(94.6)\end{array}$ & $\begin{array}{l}16 \\
(5.4)\end{array}$ & $134 / 149$ & $5 / 11$ & $52.5 \pm 16.6$ & $69.2 \pm 9.7$ & $\begin{array}{c}25 \\
(8.8)\end{array}$ & $\begin{array}{c}6 \\
(37.5)\end{array}$ & $\begin{array}{c}14 \\
(4.9)\end{array}$ & $\begin{array}{c}4 \\
(25.0)\end{array}$ & $\begin{array}{c}63 \\
(22.3)\end{array}$ & $\begin{array}{c}11 \\
(68.8)\end{array}$ & $\begin{array}{c}31 \\
(11.0)\end{array}$ & $\begin{array}{c}4 \\
(25.0)\end{array}$ & - & - \\
\hline $\begin{array}{c}\text { Wang et al., 2020* } \\
\text { [30] }\end{array}$ & - & 344 & $\begin{array}{c}211 \\
(61.3)\end{array}$ & $\begin{array}{c}133 \\
(38.7)\end{array}$ & $106 / 105$ & $59 / 74$ & $\begin{array}{c}57.0 \\
(47.0-69.0)\end{array}$ & $\begin{array}{c}70.0 \\
(62.0-77.0)\end{array}$ & $\begin{array}{c}18 \\
(8.5)\end{array}$ & $\begin{array}{c}22 \\
(16.5)\end{array}$ & - & - & $\begin{array}{c}72 \\
(34.1)\end{array}$ & $\begin{array}{c}69 \\
(51.9)\end{array}$ & $\begin{array}{c}34 \\
(16.1)\end{array}$ & $\begin{array}{c}30 \\
(22.6)\end{array}$ & - & - \\
\hline Wu et al., 2020 [31] & RCS & 84 & $\begin{array}{c}40 \\
(47.6)\end{array}$ & $\begin{array}{c}44 \\
(52.4)\end{array}$ & $9 / 31$ & $15 / 29$ & $\begin{array}{c}50.0 \\
(40.3-56.8)\end{array}$ & $\begin{array}{c}68.5 \\
(59.3-75.0)\end{array}$ & $1(2.5)$ & $4(9.1)$ & $1(2.5)$ & $4(9.1)$ & $\begin{array}{c}7 \\
(17.5)\end{array}$ & $\begin{array}{c}16 \\
(36.4)\end{array}$ & $\begin{array}{c}5 \\
(10.0)\end{array}$ & $\begin{array}{c}11 \\
(25.0)\end{array}$ & - & - \\
\hline $\begin{array}{c}\text { Ruan et al., } 2020 \\
\text { [32] }\end{array}$ & MRCS & 150 & $\begin{array}{c}82 \\
(54.7)\end{array}$ & $\begin{array}{c}68 \\
(45.3)\end{array}$ & $29 / 53$ & $19 / 49$ & $\begin{array}{c}50.0 \\
(44.0-81.0)\end{array}$ & $\begin{array}{c}67.0 \\
(15.0-81.0)\end{array}$ & $0(0)$ & $\begin{array}{c}13 \\
(19.1)\end{array}$ & - & - & $\begin{array}{c}23 \\
(28.0)\end{array}$ & $\begin{array}{c}29 \\
(42.6)\end{array}$ & $\begin{array}{c}13 \\
(15.9)\end{array}$ & $\begin{array}{c}12 \\
(17.6)\end{array}$ & $5(6.1)$ & $7(10.3)$ \\
\hline
\end{tabular}

${ }^{1}$ Values are expressed as mean \pm standard deviation, median (interquartile range) or number $(\%)$. Abbreviations: $\mathrm{CAD}=$ coronary artery disease, $\mathrm{CVD}=$ cardio-vascular disease, $\mathrm{F}=$ females, $\mathrm{M}=$ males, $\mathrm{MRCS}=$ multi-center retrospective cohort study, NS = non-survivors, $\mathrm{PCS}=$ prospective cohort study, $\mathrm{RCC}=$ retrospective case control, $\mathrm{RCS}=$ retrospective cohort study, RCsS r= retrospective case series, $\mathrm{S}=$ survivors, SRCS = single-centered retrospective cohort study, SROS = single-centered retrospective observational study. * They are all different homonymous authors, + range, $\ddagger$ two different cohorts from the same study. 


\subsection{Assessment of Bias}

Figure 2 shows the "risk of bias" graph. Low bias due to confounding was absent in all papers, as confounding was expected in all of them, although some of them [10,14-16,19,21-25,29,33,34] controlled for confounding through either multivariable and multivariate analysis, adjustment and stratification of patients. Twelve papers [9-11,13,14,24,25,27-29,31,32] had low bias due to selection of participants, as all patients eligible were included in the study and were enrolled in the same short period of time. No paper had low bias for classification of interventions as intervention status was not well defined. In addition, for the bias due to deviations from intended intervention, no study had low bias mainly due to the fact that in the majority this information was missing. Two papers $[11,29]$ show low bias due to missing data because they had complete data. Fifteen papers [9-11,13,14,16,17,24-31] had low bias in measurement of the outcome. Eight studies $[16,19,23-25,30,31]$ had low bias in the reported results. No study was overall lowly biased.

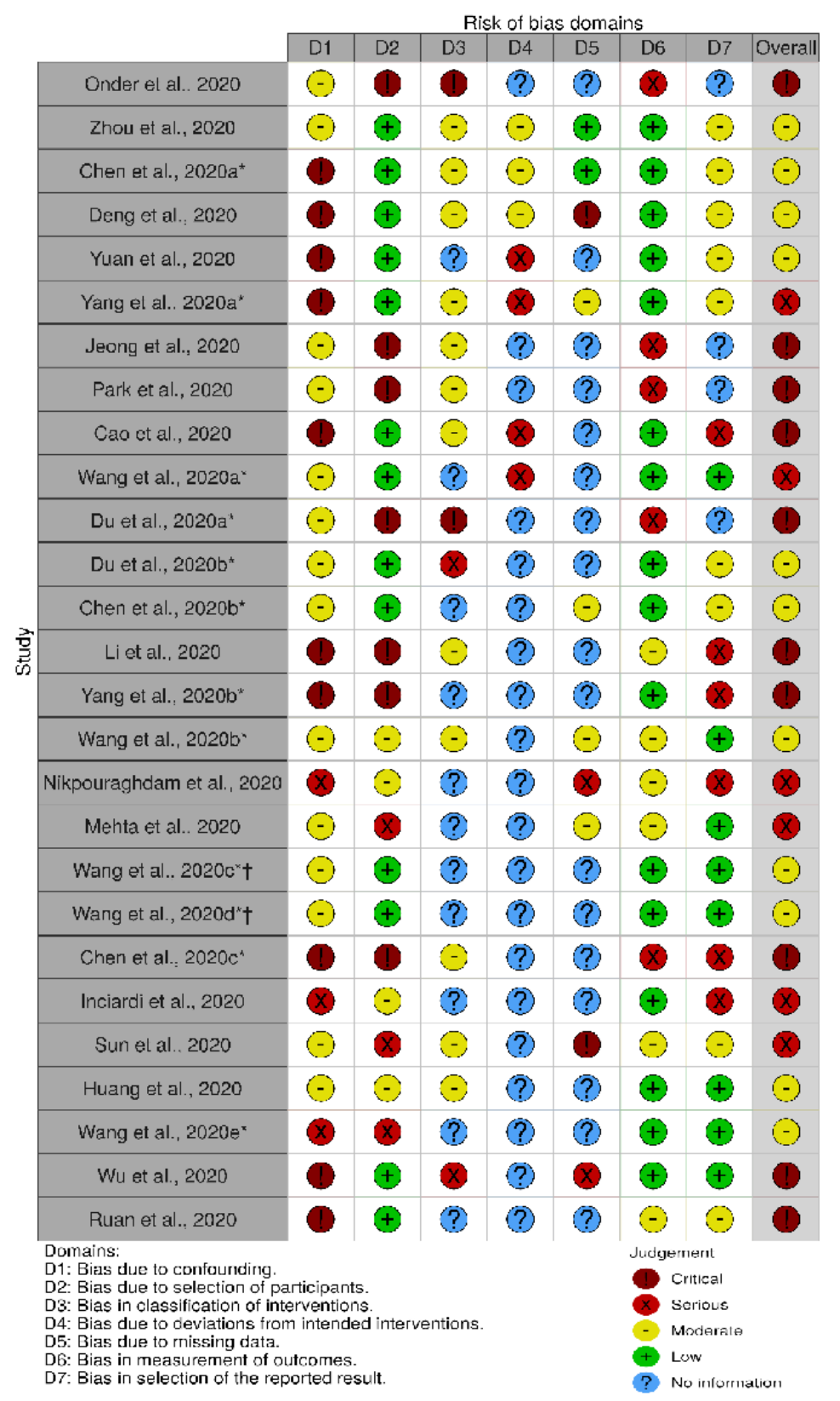

Figure 2. Risk of bias plot. * They are all different homonymous authors. † Two different cohorts from the same study. 


\subsection{Prognostic Factors for Mortality}

Incidence rate ration (IRR), heterogeneity test and Egger's test results are summarized in Table 2. The analysis revealed that men have $16 \%$ higher risk of mortality than women, as shown in Figure $3 \mathrm{~A}$ (RR: 1.16 [95\% CI: 1.05, 1.27], $p=0.003$; funnel plot in Figure S1A). As shown in Figure 3B, we found that age is another predictor of mortality, as older patients had significantly higher chance of dying than younger patients (mean difference: -15.72 [95\% CI: $-18.62,12.81], p<0.0001$; funnel plot in Figure S1B).

Furthermore, the analysis revealed that patients with CVD with CAD have a 1.96-fold [95\% CI: $1.51,2.54$ ] higher risk of mortality than patients without CVD, as presented in Figure $4 \mathrm{~A}(p<0.0001$; funnel plot in Figure S1C). In particular, the presence of CAD increased the risk of mortality by 1.90 -fold [95\% CI: 1.32, 2.74] when compared to other CVDs, as described in Figure 4B ( $p=0.0005$; funnel plot in Figure S1D). However, by conducting an analysis on CVD excluding CAD we found that the presence of other CVDs is a strong predictor of death, since patients with CVD (excluding CAD) had 2.03-fold [95\% CI: 1.41, 2.92] higher risk of mortality compared to patients without CVD (Figure 4C, $p=0.0002$; funnel plot in Figure S1E). We found that non-survivors were more likely affected by hypertension, having a 1.73-fold greater risk of mortality than patients without hypertension (Figure 4D, RR: 1.73 [95\% CI: 1.37, 2.19], $p<0.0001$; funnel plot in Figure S1F). Patients with pre-existing cerebrovascular disease tend to die 1.76-fold more than patients without cerebrovascular disease, suggesting that cerebrovascular disease is a strong predictor of death (Figure 4E, RR: 1.76 [95\% CI: 1.25, 2.50], $p<0.0001$; funnel plot in Figure S1G). Similarly, as pictured in Figure 4F, patients with diabetes had 1.59-fold higher chance of dying than patients without diabetes (RR: 1.59 [95\% CI: 1.25, 2.02], $p<0.0001$; funnel plot in Figure S1H). 
Table 2. Incidence rate ration (IRR), heterogeneity test and Egger's test results ${ }^{1}$.

\begin{tabular}{|c|c|c|c|c|c|c|c|c|}
\hline & \multicolumn{2}{|c|}{ Mean Difference } & \multicolumn{2}{|c|}{ Relative Risk } & \multicolumn{2}{|c|}{ Heterogeneity } & \multicolumn{2}{|l|}{ Publication Bias } \\
\hline & MD $[95 \% \mathrm{CI}]$ & $p$-Value & $\mathrm{RR}[95 \% \mathrm{CI}]$ & $p$-Value & $I^{2}(\%)$ & $p$-Value & Egger's Intercept [95\% CI] & $p$-Value \\
\hline Sex & NA & NA & $1.16[1.05,1.27]$ & 0.003 & 90.79 & $<0.0001$ & $2.67[-0.01,0.03]$ & 0.002 \\
\hline Age & $-15.72[-18.62,12.81]$ & $<0.0001$ & NA & NA & 86.74 & $<0.0001$ & $-6.44[-12.05,0.81]$ & 0.00 \\
\hline CVD with CAD & NA & NA & $1.96[1.51,2.54]$ & $<0.0001$ & 97.87 & $<0.0001$ & $-0.03[-0.06,0.01]$ & 0.00 \\
\hline CAD & NA & NA & $1.90[1.32,2.74]$ & 0.0005 & 97.79 & $<0.0001$ & $-0.02[-0.06,0.01]$ & 0.00 \\
\hline CVD without CAD & NA & NA & $2.03[1.41,2.92]$ & 0.0002 & 93.25 & $<0.0001$ & $-0.11[-0.34,0.13]$ & 0.00 \\
\hline Hypertension & NA & NA & $1.73[1.37,2.19]$ & $<0.0001$ & 98.01 & $<0.0001$ & $-0.08[-0.13,0.03]$ & 0.00 \\
\hline Cerebrovascular diseases & NA & NA & $1.76[1.25,2.50]$ & $<0.0001$ & 98.01 & $<0.0001$ & $-0.07[-0.16,0.01]$ & 0.01 \\
\hline Diabetes & NA & NA & $1.59[1.25,2.02]$ & $<0.0001$ & 98.71 & $<0.0001$ & $-0.02[-0.04,0.00]$ & 0.00 \\
\hline
\end{tabular}

${ }^{1}$ Abbreviations: $\mathrm{CAD}=$ coronary artery disease, $\mathrm{CI}=$ confidence interval, $\mathrm{CVD}=$ cardiovascular disease, $\mathrm{MD}=$ mean difference, $\mathrm{NA}=$ not applicable, $\mathrm{RR}=$ relative risk 

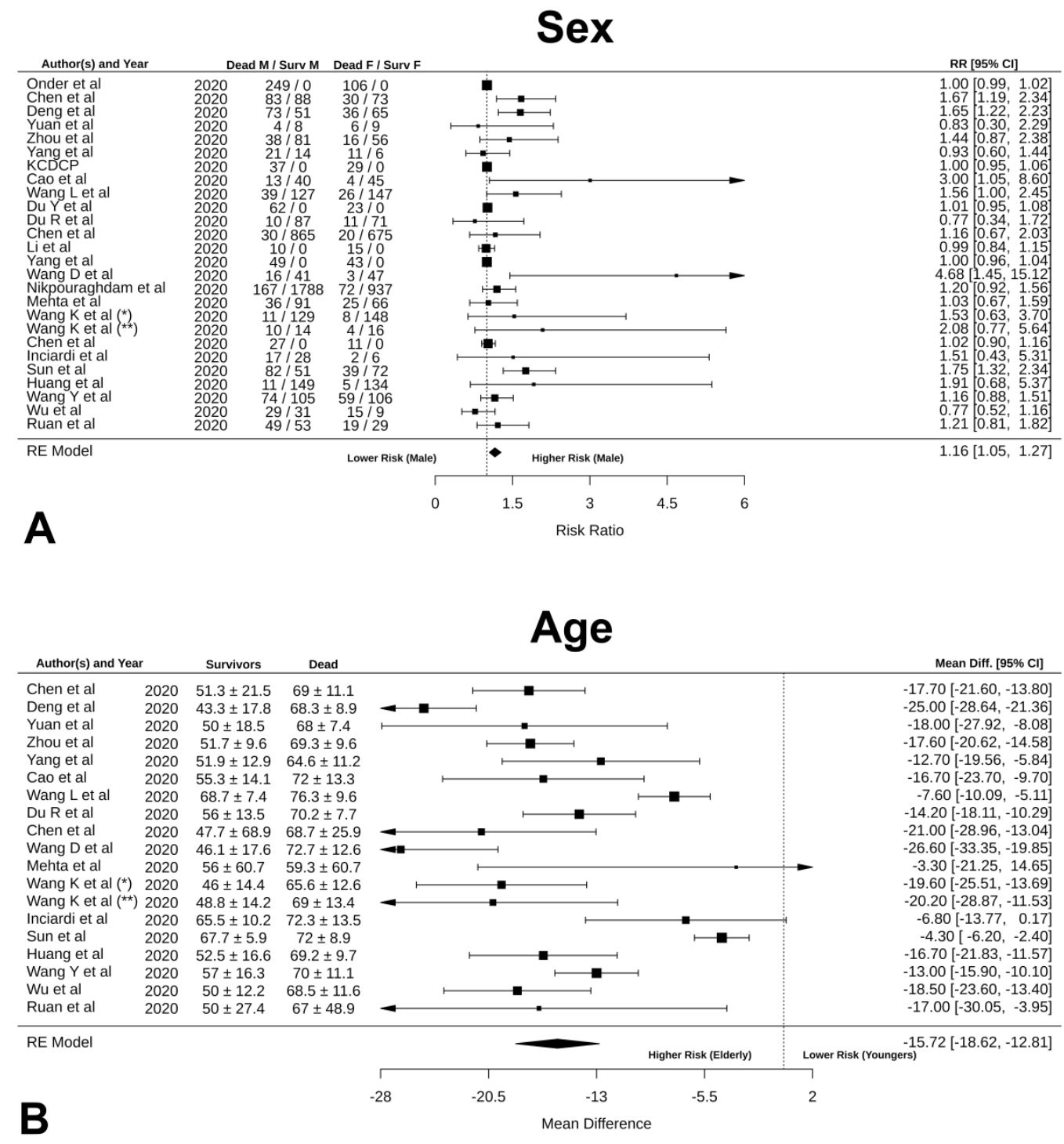

Figure 3. Forrest plot of (A) sex; (B) age. 


\section{CVD with CAD}

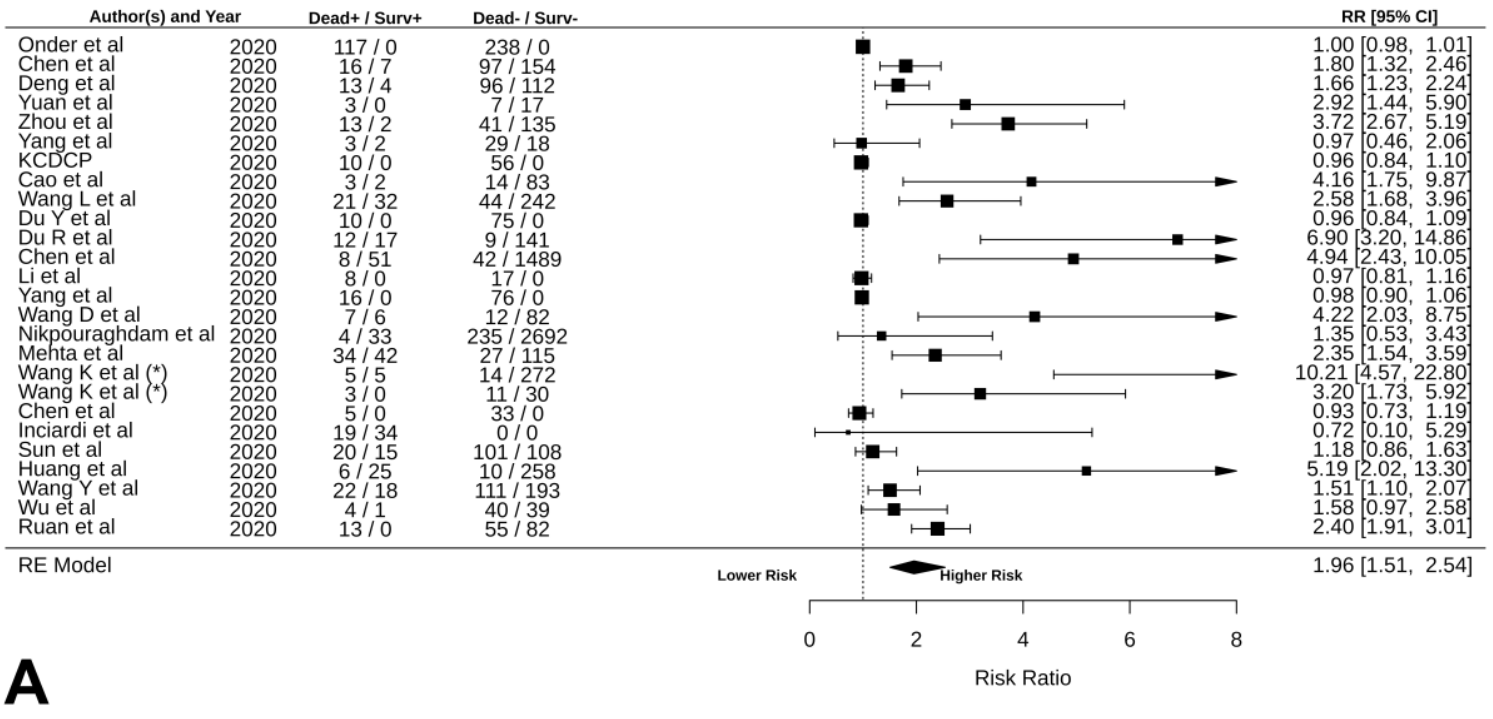

\section{CAD}

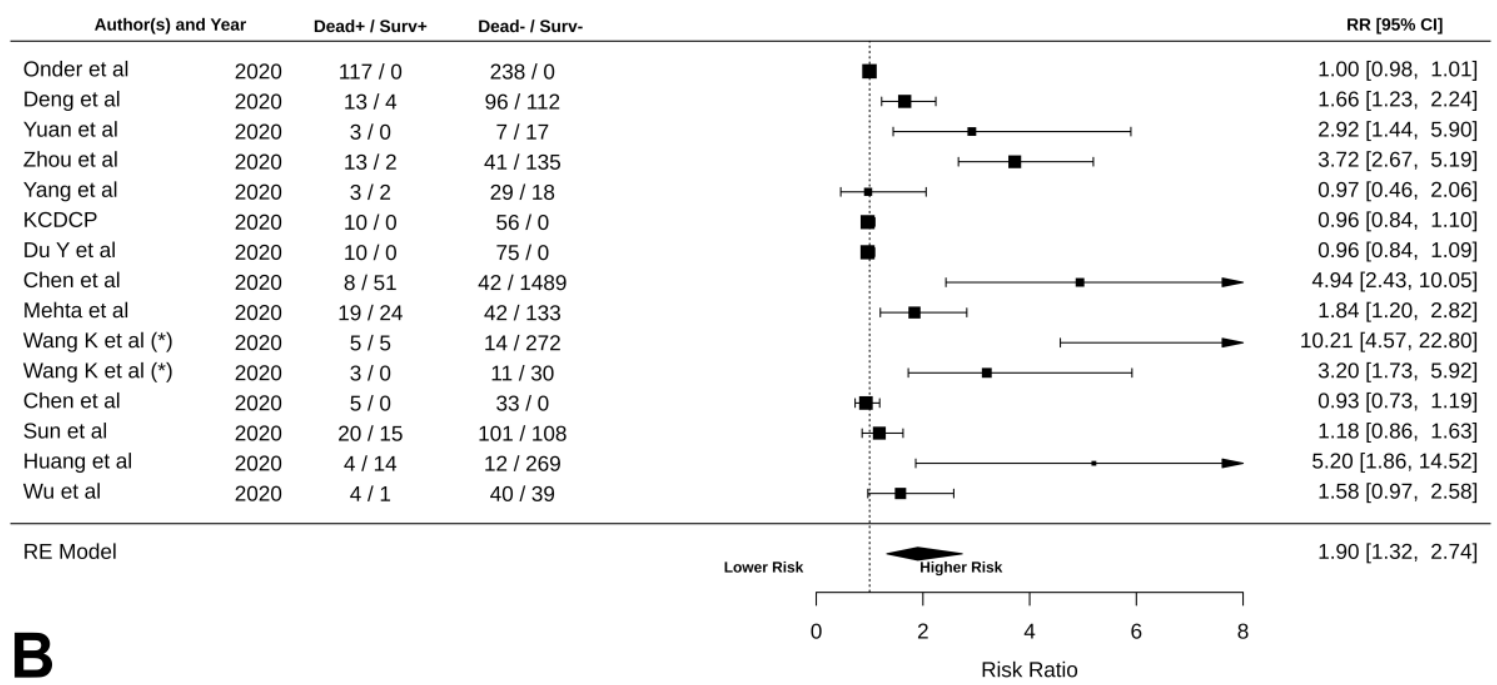

Figure 4. Cont. 


\section{CVD without CAD}

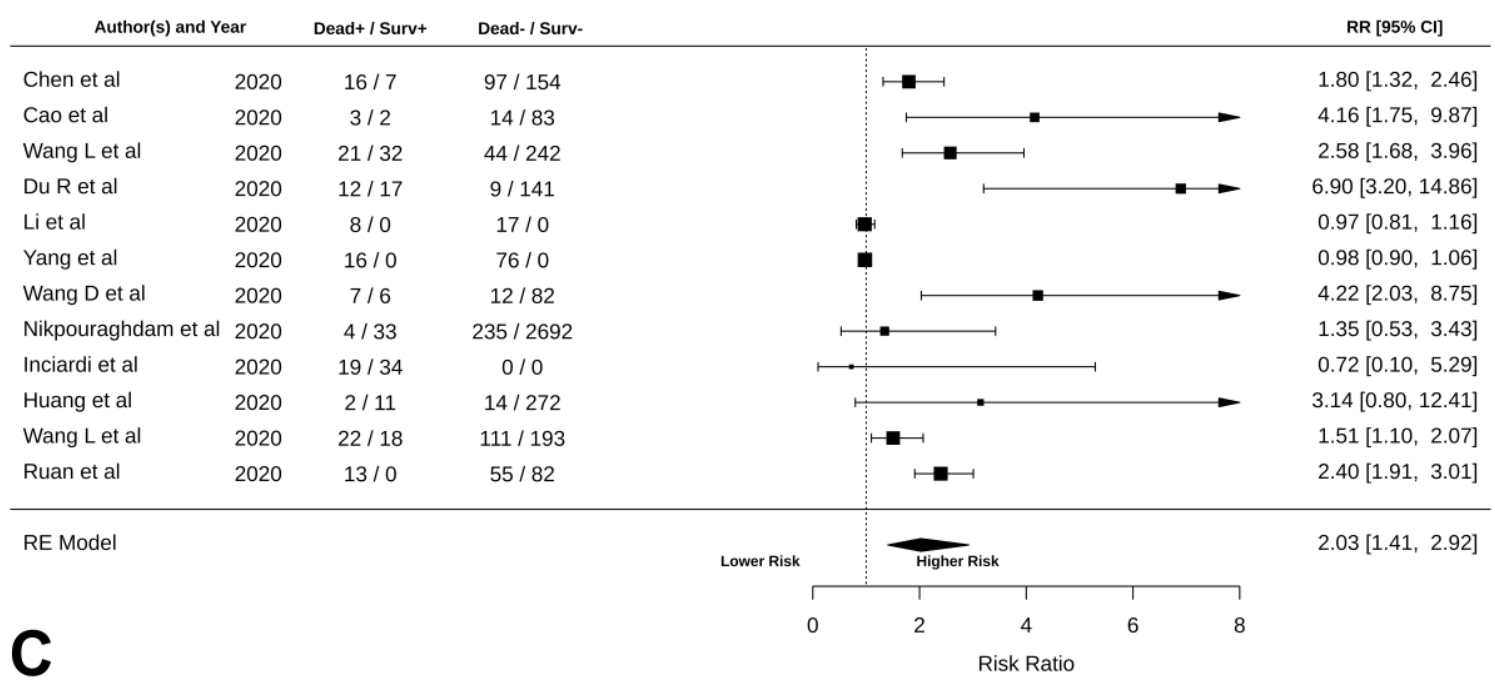

\section{Hypertension}

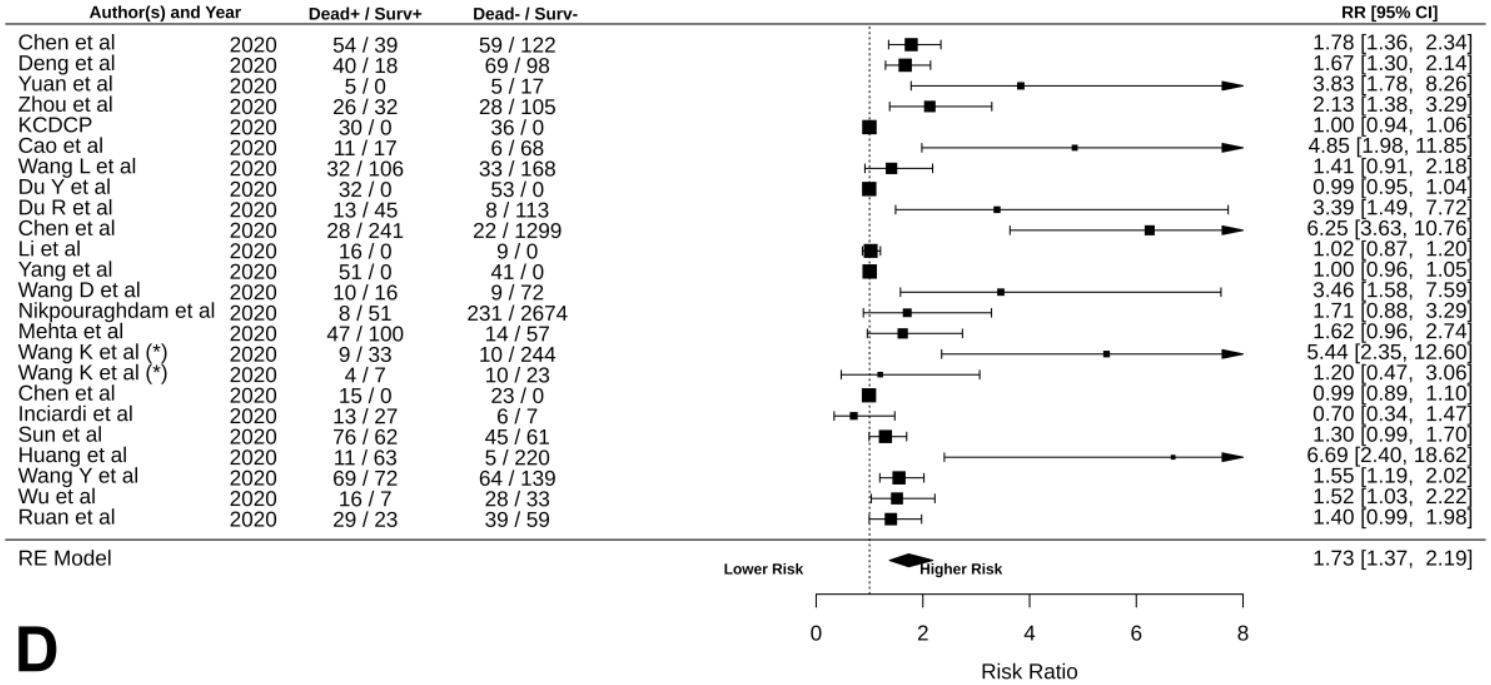

Figure 4. Cont. 


\section{Cerebrovascular Disease}

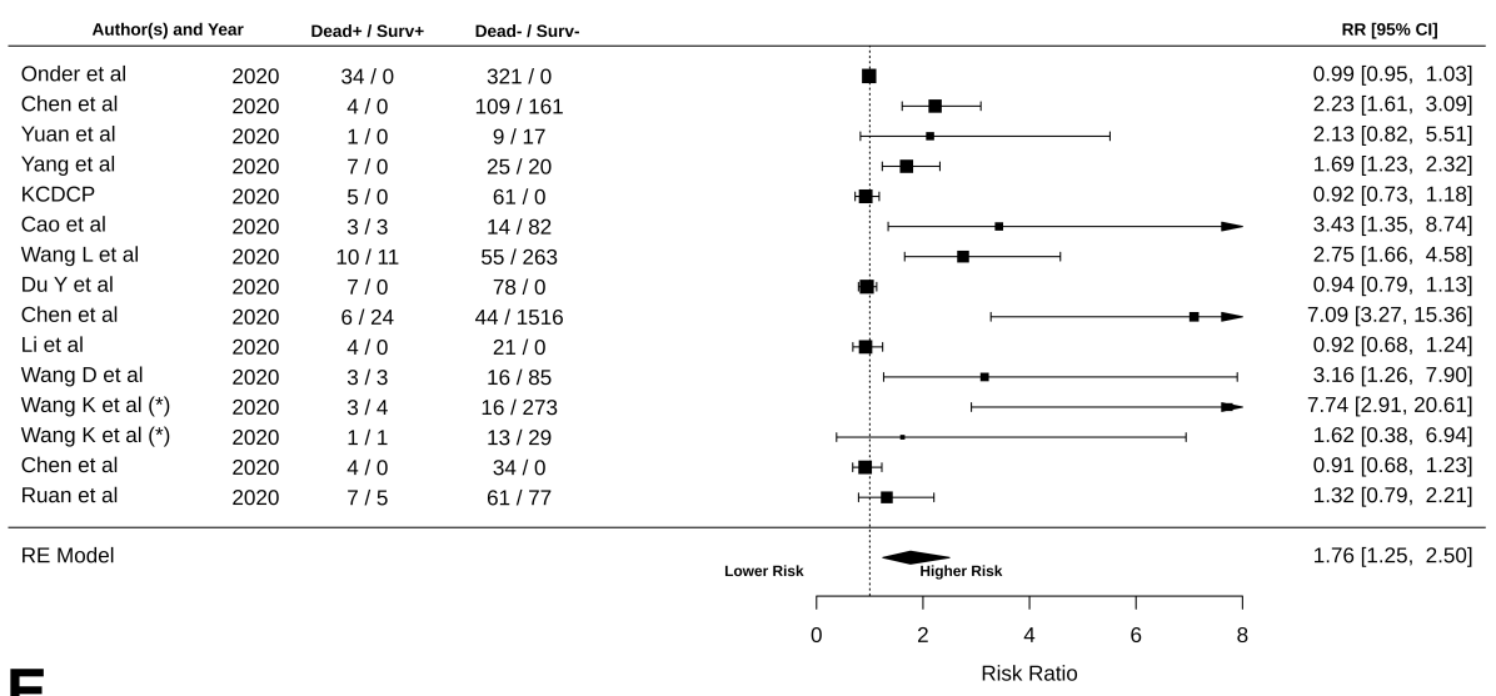

E

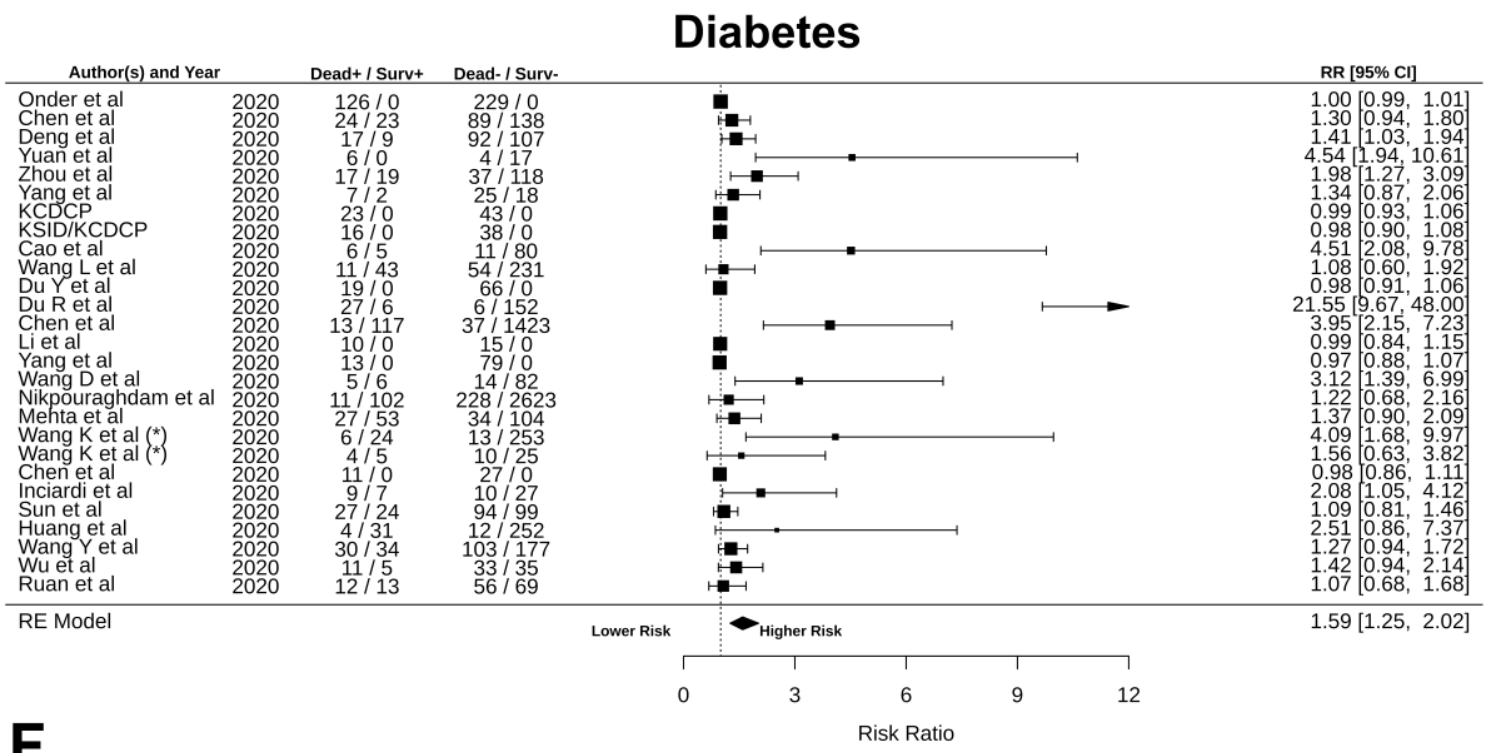

Figure 4. (A) CVD + CAD vs. patients without CVD; (B) patients with CAD vs. patients without CAD; (C) patients with CVD without CAD vs. patients without CVD; (D) patients with hypertension vs. patients without hypertension; (E) patients with cerebrovascular diseases vs. patients without cerebrovascular diseases; (F) patients with diabetes mellitus vs. patients without diabetes mellitus.

\section{Discussion}

\subsection{Increased Mortality in Males and Elderlies}

In our analysis, we quantified the risk of death in almost 8500 COVID-19 patients in relation to sex, age, pre-existing CVD and cardiovascular risk factors. To the best of our knowledge, this has not been previously done, especially in cohorts encompassing large numbers of patients. Interestingly, our updated report shows that men still are more prone to dying but the effective increase in risk in males compared to females is lower than previously reported (around 16\%). An increased risk of mortality for male COVID-19 patients (2.4 times that of women) has been widely reported. This disproportionate death ratio in men was explained by a higher incidence of pre-existing disease, higher risk behaviors, 
occupational exposure, high levels of androgens in men, and behavioral/social differences that favor women [35]. Androgens increase the expression of the transmembrane protease serine 2 (TMPRSS2) [36]. TMPRSS2 is a critical protease that enables the entry of SARS-CoV-2 in angiotensin-converting enzyme 2 (ACE2) receptors, explaining why men tend to die more from COVID-19 [36,37]. A treatment against androgens that could theoretically interfere with the course of the disease is still debated [36]. However, it must be considered that although higher male-to-female death ratio was confirmed in all the countries with available data, the United States with the largest reported outbreak of COVID-19 in the world provided only partial sex-disaggregated data and this might have biased the overall estimation of sex-related risk distributions. This warrants a careful epidemiological analysis to assess whether there has really been a turn in sex-specific differences with a rising incidence of death in women [38].

In contrast, the association with age was confirmed, with older patients being more vulnerable to die from COVID-19 [39]. Primarily responsible for the increased age-related susceptibility are the ACE2 receptors and CD26; both overexpressed in senescent cells. Both ACE2 receptors and CD26 are targets for coronaviruses, and their overexpression in older patients might mediate the increased fatality rate in COVID-19 patients [40]. ACE2 is abundantly distributed in the lungs but also in the heart, kidneys, guts, and the pancreas [41]. ACE2 is pivotal for the entry mechanism of the SARS-CoV-2 as it is harnessed by the virus as an entry point, whereas CD26 interacts with the S1 domain of the virus affecting virulence [42-44]. Another mechanism contributing to the increased mortality in elderly patients is immunosenescence, in which naïve $\mathrm{T}$ and $\mathrm{B}$ cells are produced in lower quantities, and dendritic cells do not effectively differentiate after T cell interaction [39].

\subsection{Increased Mortality in Patients with Pre-Existing CVD}

The third finding of our meta-analysis is that the presence of cardiovascular diseases, is associated with a higher risk of mortality when compared to COVID-19 patients without pre-existing CVD. Our outcomes are in contrast with the results of a previous meta-analysis conducted on three studies, which found no correlation between the history of CVD and mortality but revealed an association between CVD and enhanced disease severity [5]. It is possible that such a discrepancy could be due to the difference in terms of number of studies included in the analysis. Previous literature already suggests that CVD might be involved in promoting death in COVID-19 patients [45]. Another recent meta-analysis conducted on six papers reported that among COVID-19 patients admitted to the intensive care unit, $17.1 \%$ had hypertension and $16.4 \%$ cardiac and/or cerebrovascular diseases [3]. Wu et al. [46] also reported that patients with CVD, hypertension and diabetes tend to die more often. The cause of such an association might be complex and multifactorial. Cardiopathic patients with ventricular hypertrophy, diastolic dysfunction and heart failure tend to develop acute pulmonary hypertension while being affected by COVID-19. This can result in pulmonary edema [41]. If SARS-CoV-2 causes sepsis, then acute respiratory distress syndrome (ARDS) can occur which per se aggravates the edema, and can become the cause of death in these patients [41,47]. Additionally, when infection by SARS-CoV-2 occurs, the virus is internalized and this triggers the activation of ADAM metallopeptidase domain 17 (ADAM17). ADAM17 causes cleavage of the ACE2 receptors making them unresponsive to the negative feedback exerted by the activation of the renin-angiotensin-aldosterone system. This is ultimately responsible for further production of cytokines, which aggravate the inflammation [48]. In the presence of pre-existing CVD, the cytokine storm can exacerbate underlining diseases by aggravating pre-existing heart failure, causing depression of myocardial activity, increasing the oxygen demand/supply ratio and endothelial dysfunction [22,48]. In addition, 17\% patients with COVID-19 had pre-existing CAD and this raises the risk of death, especially when it is associated with potential hypercoagulability deriving from the febrile state [41]. Nonetheless, in our analysis, CVD with or without CAD showed very close RRs of death. In other words, although the presence of CAD alone raised the risk of death by $90 \%$, the presence of coronary disease did not increase death RR of patients with other CVDs that increases the risk > 100\%. Moreover, we have found that COVID-19 patients with hypertension had a 73\% higher RR than those without high blood pressure. Unfortunately, due to 
the lack of specific information, it was not possible to compare subgroups to study the true incremental risk associated with hypertension in COVID-19 patients with CVD. However, it has been proposed that when patients with heart failure and hypertension receive ACE inhibitors and type-I receptor blockers (ARBs), these agents contribute to the upregulation of ACE2 receptors. This increases susceptibility to contracting COVID-19. The mechanisms mentioned help explain their vulnerability to mortality. For this reason, some authors have suggested the use of alternative antihypertensive medication during the pandemic, such as calcium channel blockers [49].

\subsection{Increased Mortality in Patients with Diabetes}

The fourth finding of our analysis is the increased fatality rate in patients with diabetes. According to the meta-analysis conducted by $\mathrm{Li}$ et al. [3], patients with diabetes represented $9.7 \%$ of the COVID-19 patients in Intensive Care Unit (ICU). Susceptibility of diabetic patients towards COVID-19 and their increased chance of dying derives from the overexpression of ACE2, impaired innate immunity and delayed Th1 cell-mediated responses. These factors predispose to cytokine storm, with adverse outcomes. Furthermore, while on one hand, insulin reduces ACE2 expression, on the other, hypoglycemic drugs and statins upregulate ACE2 [50]. In addition, diabetic patients might need additional administration of insulin or secretagogues, as the viral infection can stimulate cortisol release and thus increase of blood glucose levels. However, these drugs alter water and sodium reabsorption and increase the risk of developing pulmonary edema in cardiopathic patients, especially if sepsis causes renal dysfunction. Therefore, intravenous fluids administration should also receive attention by clinicians. In this situation, concomitant treatment with ACE inhibitors can aggravate the load on the respiratory system. For this reason, some authors suggest careful evaluation of the status of the lungs and interruption of ACE inhibitors and ARBs, if necessary before ARDS manifests worsening the prognosis of patients [41].

\section{Study Limitations}

This study has some limitations. First, the majority of the studies were retrospective, predisposing to the risk of bias. Second, some of the studies included were analyses of fatality cases. Third, heterogeneity between studies was high in all the endpoints analyzed, because of a great variety in baseline characteristics. Fourth, it is possible that the definition of CVD could be different in the different hospital settings/countries, with most of the papers that were included not including detailed description of the type of cardiovascular disease of the patient. Fifth, it would have been of great interest to study the interaction between CVD and single risk factors in predicting death. Unfortunately, within the papers data were not split into sub-groups to allow these analyses. Finally, in the reports there was reported age cut-off, therefore it was not possible to examine the RR increase with age. The only data attainable was the difference in age between survivors and not survivors.

\section{Conclusions}

Our results provide a quantification of mortality risk in COVID-19 patients with pre-existing cardiovascular comorbidities. Our results demonstrate that sex, age, presence of CAD and/or other types of CVD, hypertension, cerebrovascular diseases and diabetes mellitus increase mortality in patients with COVID-19. In particular, CAD and/or other types of CVD, hypertension, cerebrovascular diseases almost double the risk of mortality. Further research to identify the underlining mechanisms of such an association is warranted. 
Supplementary Materials: The following are available online at http://www.mdpi.com/2077-0383/9/9/2685/s1, Figure S1: funnel plot of (A) sex; (B) age; (C) overall CVD (CVD + CAD) vs. patients without CVD; (D) patients with CAD vs. patients without CAD; (E) patients with CVD (and no CAD) vs. patients without CVD-CAD; (F) patients with hypertension vs. patients without hypertension; $(G)$ patients with cerebrovascular diseases vs. patients without cerebrovascular diseases; and $(\mathrm{H})$ patients with diabetes mellitus vs. patients without with diabetes mellitus.

Funding: This research received no external funding.

Acknowledgments: We thank Judith Wilson for English editing of the manuscript.

Conflicts of Interest: The authors declare no conflict of interest.

\section{References}

1. Shereen, M.A.; Khan, S.; Kazmi, A.; Bashir, N.; Siddique, R. COVID-19 infection: Origin, transmission, and characteristics of human coronaviruses. J. Adv. Res. 2020, 24, 91-98. [CrossRef] [PubMed]

2. Carter, P.; Anderson, M.; Mossialos, E. Health system, public health, and economic implications of managing COVID-19 from a cardiovascular perspective. Eur. Heart J. 2020, 41, 2516-2518. [CrossRef] [PubMed]

3. Li, B.; Yang, J.; Zhao, F.; Zhi, L.; Wang, X.; Liu, L.; Bi, Z.; Zhao, Y. Prevalence and impact of cardiovascular metabolic diseases on COVID-19 in China. Clin. Res. Cardiol. 2020, 109, 531-538. [CrossRef] [PubMed]

4. Clerkin, K.J.; Fried, J.A.; Raikhelkar, J.; Sayer, G.; Griffin, J.M.; Masoumi, A.; Jain, S.S.; Burkhoff, D.; Kumaraiah, D.; Rabbani, L.; et al. COVID-19 and Cardiovascular Disease. Circulation 2020, 141, 1648-1655. [CrossRef]

5. Aggarwal, G.; Cheruiyot, I.; Aggarwal, S.; Wong, J.; Lippi, G.; Lavie, C.J.; Henry, B.M.; Sanchis-Gomar, F. Association of Cardiovascular Disease With Coronavirus Disease 2019 (COVID-19) Severity: A Meta-Analysis. Curr. Probl. Cardiol. 2020, 45, 100617. [CrossRef]

6. Hutton, B.; Salanti, G.; Caldwell, D.M.; Chaimani, A.; Schmid, C.H.; Cameron, C.; Ioannidis, J.P.A.; Straus, S.; Thorlund, K.; Jansen, J.P.; et al. The PRISMA Extension Statement for Reporting of Systematic Reviews Incorporating Network Meta-analyses of Health Care Interventions: Checklist and Explanations. Ann. Intern. Med. 2015, 162, 777-784. [CrossRef]

7. Cumpston, M.; Li, T.; Page, M.J.; Chandler, J.; A Welch, V.; Higgins, J.P.; Thomas, J. Updated guidance for trusted systematic reviews: A new edition of the Cochrane Handbook for Systematic Reviews of Interventions. Cochrane Database Syst. Rev. 2019, 10, ED000142. [CrossRef]

8. McGuinness, L.A.; Higgins, J.P.T. Risk-of-bias VISualization (robvis): An R package and Shiny web app for visualizing risk-of-bias assessments. Res. Synth. Methods 2020, 1-7. [CrossRef]

9. Cao, J.; Hu, X.; Cheng, W.; Yu, L.; Tu, W.; Liu, Q. Correction to: Clinical features and short-term outcomes of 18 patients with corona virus disease 2019 in intensive care unit. Intensiv. Care Med. 2020, 46, 1298. [CrossRef]

10. Chen, R.; Liang, W.; Jiang, M.; Guan, W.; Zhan, C.; Wang, T.; Tang, C.; Sang, L.; Liu, J.; Ni, Z.; et al. Risk Factors of Fatal Outcome in Hospitalized Subjects With Coronavirus Disease 2019 From a Nationwide Analysis in China. Chest 2020, 158, 97-105. [CrossRef]

11. Chen, T.; Wu, D.; Chen, H.; Yan, W.; Yang, D.; Chen, G.; Ma, K.; Xu, D.; Yu, H.; Wang, H.; et al. Clinical characteristics of 113 deceased patients with coronavirus disease 2019: Retrospective study. BMJ 2020, 368, m1091. [CrossRef] [PubMed]

12. Chen, Y.; Zhao, M.; Wu, Y.; Zang, S. Epidemiological analysis of the early 38 fatalities in Hubei, China, of the coronavirus disease 2019. J. Glob. Health 2020, 10, 011004. [CrossRef]

13. Deng, Y.; Liu, W.; Liu, K.; Fang, Y.-Y.; Shang, J.; Zhou, L.; Wang, K.; Leng, F.; Wei, S.; Chen, L.; et al. Clinical characteristics of fatal and recovered cases of coronavirus disease 2019 in Wuhan, China: A retrospective study. Chin. Med J. 2020, 133, 1261-1267. [CrossRef] [PubMed]

14. Du, R.-H.; Liang, L.-R.; Yang, C.-Q.; Wang, W.; Cao, T.-Z.; Li, M.; Guo, G.-Y.; Du, J.; Zheng, C.-L.; Zhu, Q.; et al. Predictors of mortality for patients with COVID-19 pneumonia caused by SARS-CoV-2: A prospective cohort study. Eur. Respir. J. 2020, 55, 2000524. [CrossRef] [PubMed]

15. Du, Y.; Tu, L.; Zhu, P.; Mu, M.; Wang, R.; Yang, P.; Wang, X.; Hu, C.; Ping, R.; Hu, P.; et al. Clinical Features of 85 Fatal Cases of COVID-19 from Wuhan: A Retrospective Observational Study. SSRN Electron. J. 2020, 201, 1372-1379. [CrossRef] 
16. Huang, J.; Cheng, A.; Kumar, R.; Fang, Y.; Chen, G.; Zhu, Y.; Lin, S. Hypoalbuminemia predicts the outcome of COVID-19 independent of age and co-morbidity. J. Med. Virol. 2020. [CrossRef]

17. Inciardi, R.M.; Adamo, M.; Lupi, L.; Cani, D.S.; Di Pasquale, M.; Tomasoni, D.; Italia, L.; Zaccone, G.; Tedino, C.; Fabbricatore, D.; et al. Characteristics and outcomes of patients hospitalized for COVID-19 and cardiac disease in Northern Italy. Eur. Heart J. 2020, 41, 1821-1829. [CrossRef]

18. Li, X.; Wang, L.; Yan, S.; Yang, F.; Xiang, L.; Zhu, J.; Shen, B.; Gong, Z. Clinical characteristics of 25 death cases with COVID-19: A retrospective review of medical records in a single medical center, Wuhan, China. Int. J. Infect. Dis. 2020, 94, 128-132. [CrossRef]

19. Mehta, V.; Goel, S.; Kabarriti, R.; Cole, D.; Goldfinger, M.; Acuna-Villaorduna, A.; Pradhan, K.; Thota, R.; Reissman, S.; Sparano, J.A.; et al. Case Fatality Rate of Cancer Patients with COVID-19 in a New York Hospital System. Cancer Discov. 2020, 10, 935-941. [CrossRef]

20. Nikpouraghdam, M.; Farahani, A.J.; Alishiri, G.; Heydari, S.; Ebrahimnia, M.; Samadinia, H.; Sepandi, M.; Jafari, N.J.; Izadi, M.; Qazvini, A.; et al. Epidemiological characteristics of coronavirus disease 2019 (COVID-19) patients in IRAN: A single center study. J. Clin. Virol. 2020, 127, 104378. [CrossRef]

21. Onder, G.; Rezza, G.; Brusaferro, S. Case-Fatality Rate and Characteristics of Patients Dying in Relation to COVID-19 in Italy. JAMA 2020, 323, 2763667. [CrossRef] [PubMed]

22. Sun, H.; Ning, R.; Tao, Y.; Yu, C.; Deng, X.; Zhao, C.; Meng, S.; Tang, F.; Xu, D. Risk Factors for Mortality in 244 Older Adults With COVID-19 in Wuhan, China: A Retrospective Study. J. Am. Geriatr. Soc. 2020, 68, E19-E23. [CrossRef] [PubMed]

23. Wang, D.; Yin, Y.; Hu, C.; Liu, X.; Zhang, X.; Zhou, S.; Jian, M.; Xu, H.; Prowle, J.R.; Hu, B.; et al. Clinical course and outcome of 107 patients infected with the novel coronavirus, SARS-CoV-2, discharged from two hospitals in Wuhan, China. Crit. Care 2020, 24, 1-9. [CrossRef] [PubMed]

24. Wang, K.; Zuo, P.; Liu, Y.; Zhang, M.; Zhao, X.; Xie, S.; Zhang, H.; Chen, X.; Liu, C. Clinical and laboratory predictors of in-hospital mortality in patients with COVID-19: A cohort study in Wuhan, China. Clin. Infect. Dis. 2020. [CrossRef]

25. Wang, L.; He, W.; Yu, X.; Hu, D.; Bao, M.; Liu, H.; Zhou, J.; Jiang, H. Coronavirus disease 2019 in elderly patients: Characteristics and prognostic factors based on 4-week follow-up. J. Infect. 2020, 80, 639-645. [CrossRef]

26. Yang, F.; Shi, S.; Zhu, J.; Shi, J.; Dai, K.; Chen, X. Analysis of 92 deceased patients with COVID-19. J. Med. Virol. 2020. [CrossRef]

27. Yang, X.; Yu, Y.; Xu, J.; Shu, H.; Xia, J.; Liu, H.; Wu, Y.; Zhang, L.; Yu, Z.; Fang, M.; et al. Clinical course and outcomes of critically ill patients with SARS-CoV-2 pneumonia in Wuhan, China: A single-centered, retrospective, observational study. Lancet Respir. Med. 2020, 8, 475-481. [CrossRef]

28. Yuan, M.; Yin, W.; Tao, Z.; Tan, W.; Hu, Y. Association of radiologic findings with mortality of patients infected with 2019 novel coronavirus in Wuhan, China. PLoS ONE 2020, 15, e0230548. [CrossRef]

29. Zhou, F.; Yu, T.; Du, R.; Fan, G.; Liu, Y.; Liu, Z.; Xiang, J.; Wang, Y.; Song, B.; Gu, X.; et al. Clinical course and risk factors for mortality of adult inpatients with COVID-19 in Wuhan, China: A retrospective cohort study. Lancet 2020, 395, 1054-1062. [CrossRef]

30. Wang, Y.; Lu, X.; Li, Y.; Chen, H.; Chen, T.; Su, N.; Huang, F.; Zhou, J.; Zhang, B.; Yan, F.; et al. Clinical Course and Outcomes of 344 Intensive Care Patients with COVID-19. Am. J. Respir. Crit. Care Med. 2020, 201, 1430-1434. [CrossRef]

31. Wu, C.; Chen, X.; Cai, Y.; Xia, J.; Zhou, X.; Xu, S.; Huang, H.; Zhang, L.; Zhou, X.; Du, C.; et al. Risk Factors Associated With Acute Respiratory Distress Syndrome and Death in Patients With Coronavirus Disease 2019 Pneumonia in Wuhan, China. JAMA Intern. Med. 2020, 180, 934-943. [CrossRef]

32. Ruan, Q.; Yang, K.; Wang, W.; Jiang, L.; Song, J. Clinical predictors of mortality due to COVID-19 based on an analysis of data of 150 patients from Wuhan, China. Intensiv. Care Med. 2020, 46, 846-848. [CrossRef] [PubMed]

33. Jeong, E.K.; Park, O.; Park, Y.J.; Park, S.Y.; Kim, Y.M.; Kim, J.; Jo, J.; Kim, J.; Kim, T.; Kweon, S. Coronavirus disease-19: The first 7,755 cases in the Republic of Korea. Osong. Public Health Res. Perspect 2020, 11, 85-90.

34. Park, S.; Lee, M.; Kim, S.; Kwak, Y.; Kwon, K.; Park, J.; Shin, B.; Lee, K.; Kim, H.; Kim, Y. Analysis on 54 mortality cases of coronavirus disease 2019 in the Republic of Korea from January 19 to March 10, 2020. J. Korean Med. Sci. 2020, 35, e132. 
35. Jin, J.-M.; Bai, P.; He, W.; Wu, F.; Liu, X.-F.; Han, D.-M.; Liu, S.; Yang, J.-K. Gender Differences in Patients With COVID-19: Focus on Severity and Mortality. Front. Public Health 2020, 8, 152. [CrossRef] [PubMed]

36. Gebhard, C.; Regitz-Zagrosek, V.; Neuhauser, H.K.; Morgan, R.; Klein, S.L. Impact of sex and gender on COVID-19 outcomes in Europe. Biol. Sex Differ. 2020, 11, 1-13. [CrossRef] [PubMed]

37. Guzik, T.; Mohiddin, S.A.; DiMarco, A.; Patel, V.; Savvatis, K.; Marelli-Berg, F.M.; Madhur, M.S.; Tomaszewski, M.; Maffia, P.; D'Acquisto, F.; et al. COVID-19 and the cardiovascular system: Implications for risk assessment, diagnosis, and treatment options. Cardiovasc. Res. 2020, 116, 1666-1687. [CrossRef]

38. Sharma, G.; Volgman, A.S.; Michos, E.D. Sex Differences in Mortality From COVID-19 Pandemic. JACC Case Rep. 2020, 2, 1407-1410. [CrossRef]

39. Castle, S.C. Clinical Relevance of Age-Related Immune Dysfunction. Clin. Infect. Dis. 2000, 31, 578-585. [CrossRef]

40. Sargiacomo, C.; Sotgia, F.; Lisanti, M.P. COVID-19 and chronological aging: Senolytics and other anti-aging drugs for the treatment or prevention of corona virus infection? Aging 2020, 12, 6511-6517. [CrossRef]

41. Mehra, M.R.; Ruschitzka, F. COVID-19 Illness and Heart Failure. JACC: Heart Fail. 2020, 8, 512-514. [CrossRef]

42. Wu, C.; Liu, Y.; Yang, Y.; Zhang, P.; Zhong, W.; Wang, Y.; Wang, Q.; Xu, Y.; Li, M.; Li, X.; et al. Analysis of therapeutic targets for SARS-CoV-2 and discovery of potential drugs by computational methods. Acta Pharm. Sin. B 2020, 10, 766-788. [CrossRef] [PubMed]

43. Gheblawi, M.; Wang, K.; Viveiros, A.; Nguyen, Q.; Zhong, J.-C.; Turner, A.J.; Raizada, M.K.; Grant, M.B.; Oudit, G.Y. Angiotensin-Converting Enzyme 2: SARS-CoV-2 Receptor and Regulator of the Renin-Angiotensin System. Circ. Res. 2020, 126, 1456-1474. [CrossRef] [PubMed]

44. Vankadari, N.; Wilce, J.A. Emerging COVID-19 coronavirus: Glycan shield and structure prediction of spike glycoprotein and its interaction with human CD26. Emerg. Microbes Infect. 2020, 9, 601-604. [CrossRef]

45. Driggin, E.; Madhavan, M.V.; Bikdeli, B.; Chuich, T.; Laracy, J.; Biondi-Zoccai, G.; Brown, T.S.; Der Nigoghossian, C.; Zidar, D.A.; Haythe, J.; et al. Cardiovascular Considerations for Patients, Health Care Workers, and Health Systems During the COVID-19 Pandemic. J. Am. Coll. Cardiol. 2020, 75, 2352-2371. [CrossRef]

46. Wu, Z.; McGoogan, J.M. Characteristics of and Important Lessons from the Coronavirus Disease 2019 (COVID-19) Outbreak in China. JAMA 2020, 323, 1239. [CrossRef]

47. Riera, J.; Argudo, E.; Ruiz-Rodríguez, J.C.; Ferrer, R. Extracorporeal Membrane Oxygenation for Adults with Refractory Septic Shock. ASAIO J. 2019, 65, 760-768. [CrossRef]

48. Wang, K.; Gheblawi, M.; Oudit, G.Y. Angiotensin Converting Enzyme 2: A Double-Edged Sword. Circulation 2020, 142, 426-428. [CrossRef]

49. Fang, L.; Karakiulakis, G.; Roth, M. Are patients with hypertension and diabetes mellitus at increased risk for COVID-19 infection? Lancet Respir. Med. 2020, 8, e21. [CrossRef]

50. Muniyappa, R.; Gubbi, S. COVID-19 pandemic, coronaviruses, and diabetes mellitus. Am. J. Physiol. Metab. 2020, 318, E736-E741. [CrossRef]

(C) 2020 by the authors. Licensee MDPI, Basel, Switzerland. This article is an open access article distributed under the terms and conditions of the Creative Commons Attribution (CC BY) license (http://creativecommons.org/licenses/by/4.0/). 